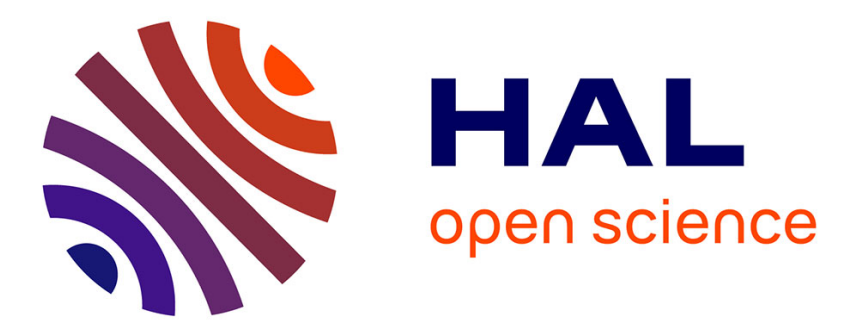

\title{
Inside/outside the brain binary cavitation localization based on the lowpass filter effect of the skull on the harmonic content: a proof of concept study
}

Guillaume Maimbourg, Alexandre Houdouin, Mathieu D. Santin, Stéphane Lehéricy, Mickaël Tanter, Jean-François Aubry

\section{To cite this version:}

Guillaume Maimbourg, Alexandre Houdouin, Mathieu D. Santin, Stéphane Lehéricy, Mickaël Tanter, et al.. Inside/outside the brain binary cavitation localization based on the lowpass filter effect of the skull on the harmonic content: a proof of concept study. Physics in Medicine and Biology, 2018, 63 (13), pp.135012. 10.1088/1361-6560/aaca21 . hal-02405927

\section{HAL Id: hal-02405927 \\ https://hal.science/hal-02405927}

Submitted on 11 Dec 2019

HAL is a multi-disciplinary open access archive for the deposit and dissemination of scientific research documents, whether they are published or not. The documents may come from teaching and research institutions in France or abroad, or from public or private research centers.
L'archive ouverte pluridisciplinaire HAL, est destinée au dépôt et à la diffusion de documents scientifiques de niveau recherche, publiés ou non, émanant des établissements d'enseignement et de recherche français ou étrangers, des laboratoires publics ou privés. 
Inside/outside the brain binary cavitation localization based on the lowpass filter effect of the skull on the harmonic content: a proof of concept study

Guillaume Maimbourg ${ }^{1,2}$, Alexandre Houdouin ${ }^{1}$, Mathieu Santin ${ }^{3,4}$, Stéphane Lehericy ${ }^{3,4}$, Mickael Tanter ${ }^{1}$ and Jean-François Aubry ${ }^{1}$

1- Institut Langevin, ESPCI Paris, CNRS UMR7587, INSERM U 979, F-75012, France

2- Université Paris Diderot, Sorbonne Paris Cité, F-75013, France

3- CENIR, ICM, F-75013, France

4- UPMC Univ. Paris 6, CNRS UMR7225, INSERM UMRS 975, Hôpital Pitié-Salpêtrière, Centre de Recherche de I'Institut du Cerveau et de la Moelle Épinière, F-75013, France

\begin{abstract}
$\underline{\text { Abstract }}$
Cavitation activity induced by ultrasound may occur during High Intensity Focused Ultrasound treatment, due to bubble nucleation under high Peak Negative Pressure, and during Blood-BrainBarrier (BBB) disruption, due to injected Ultrasound Contrast Agents (UCAs). Such microbubble activity has to be monitored to assess the safety and efficiency of ultrasonic brain treatments. In this study, we aim at assessing whether cavitation occurs within cerebral tissue by binary discriminating cavitation activity originating from the inside or the outside of the skull. Results were obtained from both in vitro experiments mimicking BBB opening, by using UCA flow, and in vitro thermal necrosis in calf brain samples. The sonication was applied using a $1 \mathrm{MHz}$ focused transducer and the acoustic response of the microbubbles was recorded with a wideband Passive Cavitation Detector (PCD). The spectral content of the recorded signal was used to localize microbubble activity. Since the skull acts as a low pass filter, the ratio of high harmonics to low harmonics is lower for cavitation events located inside the skull compared to events outside the skull. Experiments showed that the ratio of the $5 / 2$ ultraharmonic to the $1 / 2$ subharmonic for binary localization cavitation activity achieves $100 \%$ sensitivity and specificity for both monkey and human skulls. The harmonic ratio of the fourth to the second harmonic provided $100 \%$ sensitivity and $96 \%$ and $46 \%$ specificity on non human primate for thermal necrosis and BBB opening respectively. Nonetheless, the harmonic ratio remains promising for human applications, as experiments showed $100 \%$ sensitivity and $100 \%$ specificity for both thermal necrosis and BBB opening through the human skull.
\end{abstract}

\title{
Introduction
}

The skull induces strong aberrations, both in phase and amplitude, when ultrasounds (US) are transmitted through it. Consequently, a spherical phase profile does not achieve optimal focusing through the skull. For this reason, the use of ultrasound for brain treatment had only remained possible via craniotomy for a long time $[2,3]$. However, the development of adaptive focusing techniques to compensate for skull distortions has shed a new light on ultrasonic brain applications [4-6]. Moreover, previous studies have demonstrated that the phase shift assessment required for performing the adaptive focusing can be performed non-invasively with numerical simulation based on a Magnetic Resonance Imaging (MRI) [7-9] or Computed Tomography (CT) [10-13] of the skull. Two major applications using focused transcranial ultrasound are currently under fundamental and clinical investigation: Blood-Brain-Barrier (BBB) opening using focused ultrasound combined with Ultrasound Contrast Agent (UCA) injection and thermal necrosis by High Intensity Focused Ultrasound (HIFU). 
BBB opening improves drug delivery in the brain's parenchyma. Non-localized [14] and/or invasive [15, 16] openings have been carried out, but are poorly effective and potentially highly hazardous for patients $[17,18]$. For these reasons, an alternative method, combining the injection of encapsulated microbubbles with transcranial focused ultrasound, has been developed to open the BBB $[19,20]$. The microbubbles - commonly commercial UCAs - are injected intravenously and perfused throughout the body, encompassing the brain's vascular system. The UCAs are then locally excited by focused ultrasound (FUS) in the targeted volume. Previous studies have established enhancement of antitumor molecules delivery by BBB opening on small animals [21, 22]. Additionally, the feasibility of trans-skull BBB opening was demonstrated on non-human primates at lower US frequencies [23, 24]. Despite the successful BBB openings obtained with UCA+FUS, improving the cavitation activity monitoring remains essential for a safe and efficient clinical translation. Indeed, if stable cavitation is a desired phenomenon during BBB opening, inertial cavitation is not. McDannold et al. demonstrated that the acoustic signature of inertial cavitation was correlated with hypointense spots in $T 2^{*}$-weighted MR image and histological evidence of hemorrhage [1].

Furthermore, FUS can achieve brain tissue ablation by thermal necrosis. This approach has been extensively tested on animal models [25-29]. Brain tissue ablation is currently used on humans for treating neurological disorders such as essential tremors [30-33], Parkinson's diseases [34], neuropathic pain [35, 36] and drug resistant obsessive-compulsive disorder [37]. The treatment is achieved with high acoustic intensity $\left(1000 \mathrm{~W} / \mathrm{cm}^{2}\right.$ and above) and long sonication duration (10-20s) to induce a $55-57^{\circ} \mathrm{C}$ peak temperature [36] in tissues, resulting in their necrosis. The sonication can spontaneously nucleate microbubbles, which then oscillate in a stable or inertial cavitation state driven by the ultrasound beam. The inertial cavitation is known to potentially induce massive hemorrhages during HIFU procedures[38].

To ensure the safety, efficiency, and repeatability of BBB opening and thermal necrosis, monitoring the acoustic signature of microbubbles in real time is therefore crucial. Passive cavitation detector is able to quantify microbubble activity and discriminate between stable and inertial cavitation. Instantaneous and cumulative levels of cavitation can be computed, such as the stable cavitation dose (SCD) $[39,40]$ based on the harmonic level of the acoustic response, or the inertial cavitation dose (ICD) [39-43] based on the broadband level. However, no accurate and low cost method currently exists to establish whether the acoustic signal is produced from microbubbles located inside or outside the skull. Current approaches to spatially localize cavitation activity are based on passive [44-50] or active [50] multi-element arrays. But the imaging probe, required for cavitation mapping, is difficult to embed in a brain therapy device and is not used in clinics yet. Consequently, thermal ablations by FUS are interrupted as soon as the signal recorded by the PCDs exceeds a given threshold, wherever the signal originates from.

The acoustic response of microbubbles driven by ultrasound excitation at the frequency $f_{0}$ is known to be highly non-linear[51]. Consequently, the spectral content of the signal comprises harmonics $\left(2 f_{0}\right.$, $\left.3 f_{0}, 4 f_{0} \ldots\right)$, one subharmonic $\frac{1}{2} f_{0}$ and ultraharmonics $\left(\frac{3}{2} f_{0}, \frac{5}{2} f_{0}, \frac{7}{2} f_{0} \ldots\right)$. Harmonics are not specific to microbubble activity. They can be induced by the nonlinearities in power amplification or during the wave propagation in water. By contrast, the half harmonics $(1 / 2,3 / 2,5 / 2 \ldots)$ are the signature of the acoustic response of microbubbles [51]. The purpose of this study is to show that the frequencydependent attenuation of the signal passing through the skull [52] affects the microbubbles' acoustic response, and that it can be used as a low-cost indicator for binary localization of cavitation events occurring inside or outside the skull. In vitro experiments mimicking cavitation events originating from either BBB opening or thermal necrosis have been conducted. Human and monkey skull samples were used to evaluate their respective effects on the acoustic signal recorded by a PCD. 


\section{Materials and methods}

\section{Experimental setup}

An experimental setup (Fig. 2) was designed to mimic cavitation events originating from BBB opening and thermal necrosis in well-controlled and replicable conditions. The single-element transducer (Sonic Concept H-101MR, Bothell, WA, USA) used for experiments has a $59 \mathrm{~mm}$ radius of curvature, a $\mathrm{f}$-number of 1 , and is operated at $914 \mathrm{kHz}$. The focused beam propagated through water only (even in the configuration with a skull sample placed in front of the PCD: Fig. 2, left). Consequently, the sonication was independent from the presence, or absence, of the skull sample. Calibration was performed using an heterodyne laser interferometer [53] to determine the peak negative pressure (PNP) against the voltage applied to the transducer. The electrical excitation was generated by an arbitrary function generator (Tektronik AFG3101C, Beaverton, OR, USA) and amplified (Amplifier Research Model 40AD1, Souderton, PA, USA).

The PCD was a wideband transducer (Imasonic S/N 1505, Besançon, France) with the following features: $70 \mathrm{~mm}$ focal length, $14 \mathrm{~mm}$ aperture, $7.6 \mathrm{MHz}$ central frequency and a $-6 \mathrm{~dB}$ bandwidth from 4.5 $\mathrm{MHz}$ to $14.4 \mathrm{MHz}$. Figure 1 presents its bandwidth for frequency from 0 to $15 \mathrm{MHz}$. The PCD was placed confocally and almost orthogonally to the excitation beam. For experiments assessing the filtering effect of the skull on cavitation signals, a skull sample (monkey or human) was placed between the PCD and the confocal spot to mimic bubble activity from 'inside the skull'. When a skull sample was in place, a low noise amplifier (Olympus 5676, Tokyo, Japan) was added to provide a fixed $+40 \mathrm{~dB}$ amplification of the PCD signal before digitalization. The signal was then digitized into 14bits at 100 Msamples/s by an oscilloscope (TiePie HS5, Sneek, Netherlands). The transducers and the skull were immersed in water for acoustic coupling.

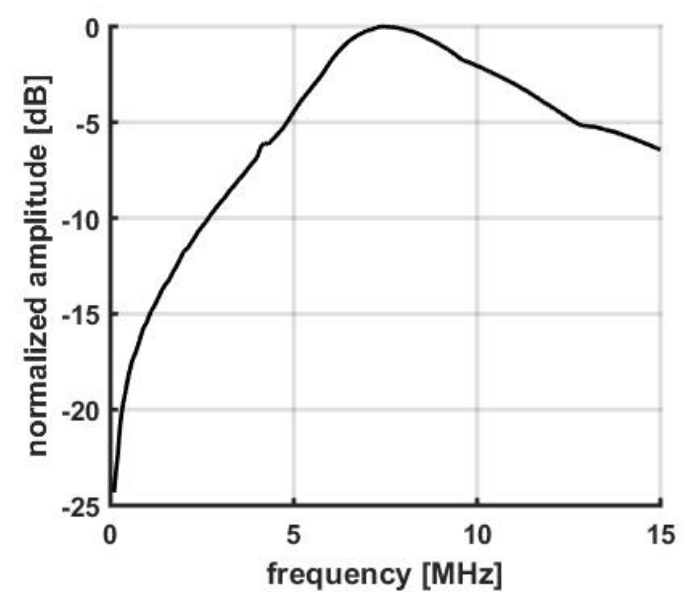

Figure 1: bandwidth (dB scale) of the PCD.

Studies were carried out on one skull sample from macaca fascicularis and six human skulls (\#1 to \#6). The PCD was located in the temporal area of the human skulls. The human skulls were provided by the Institut d'Anatomie (UFR Biomédicale des Saints-Pères Université Paris Descartes, Paris, France) and tattooed with individual numbers, as approved by the ethics committee of the Centre du Don des Corps (Université Paris Descartes, Paris, France). The donors provided informed consent before death. The skull samples were stored and kept dry. 48 hours before the experiments, the samples were immersed in water and degassed under reduced pressure $(\mathrm{P}<2 \mathrm{mbar})$. 
A bolus of UCA (SonoVue, Bracco Research SA, Switzerland) was injected with a syringe and circulated in a water closed circuit, mimicking intravenous injection and circulation in the vasculature (Fig. 2). A peristaltic pump (Watson \& Marlow 313F, Falmouth, UK) provided a continuous flow (1.4 L/min) of microbubbles in the focal acoustic volume. This device helped us maintain a steady microbubble concentration throughout the acquisition (more than 10 seconds). An acoustic window was made from a latex US probe cover (Richter Rubber Technology, Kedah, Malaysia). The confocal spot of the transducers was located in this window. An in-house expansion tank was mounted in the circuit to trap the macrobubbles that could be induced by the water circulation and to adjust water pressure. Three scenarios were investigated: 'free propagation' (no skull sample in front of the PCD), 'propagation through the monkey skull' and 'propagation through a human skull (\#1 to \#6)'. An identical protocol was followed for those three setups. A 914-kHz sinusoidal 30ms-burst was repeated every $2 \mathrm{~s}$. The input voltage of the transducer was set to obtain a 0.6 MPa PNP at the focus. This value was chosen in consideration of the results reported by McDannold et al. to induce a transient BBB opening [54]. 5 acquisitions were completed before the UCA injection (baseline) and 20 acquisitions afterwards.

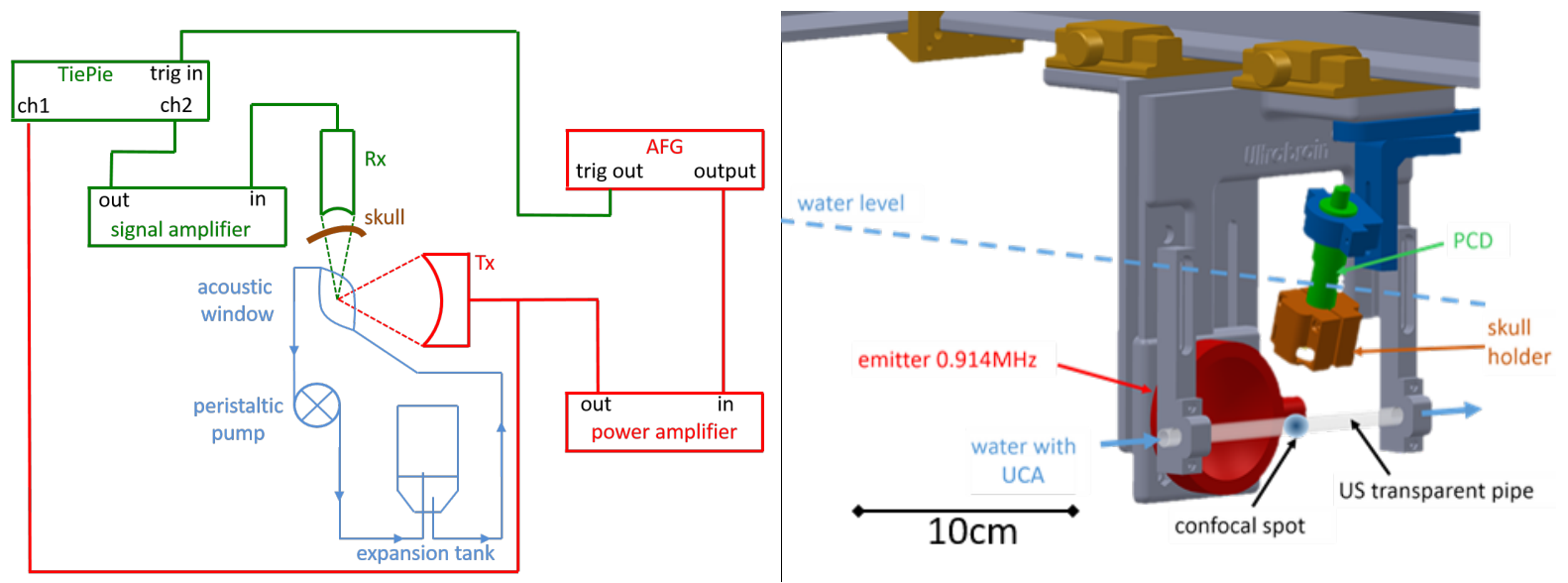

Figure 2. Left: Setup mimicking BBB opening; in red, the power circuit with the ultrasonic emitter; in green, the signal circuit with the PCD (Rx); and in blue, the hydraulic circuit for UCA circulation. Right: A 3-dimensionnal representation of the experimental setup.

\section{Thermal necrosis mimicking setup}

The goal was to study the acoustic response of microbubbles generated in situ by FUS. A fresh calf brain sample was employed to mimic thermal necrosis of the brain tissues. The sample was immersed in a saline solution and degassed for 3 hours under reduced pressure ( $P<2 \mathrm{mbar})$. Then, it was placed in a latex US probe cover (Richter Rubber Technology, Kedah, Malaysia) and positioned at the focus of the transducers with an holder (Fig. 3, left) manufactured in-house. 


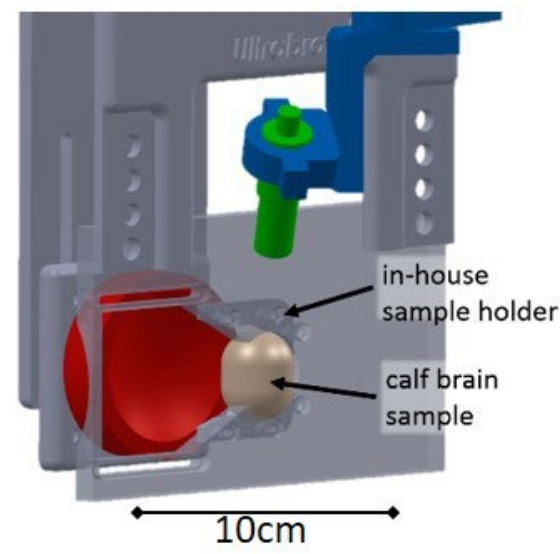

Figure 3. Detailed view of the brain sample holder. The brain sample is positioned at the confocal spot of both emitter (in red) and PCD (in green).

A $914 \mathrm{kHz}$ and 4.5 MPa PNP sinusoidal burst was emitted during 0.7s with a $6 \mathrm{~s}$ repetition time. 20 acquisitions were performed to test repeatability. Only one human skull (sample \#1) was tested for thermal necrosis.

\section{Data analysis}

Power spectra of the PCD recordings were obtained by numerical Fast Fourier Transform (FFT) with Matlab (Mathworks, Natick, MA, USA). The power spectra were deconvoluted by the bandwidth of the $P C D$ and then normalized by the maximum value obtained for the fundamental frequency. Therefore, all the results below are independent of the PCD bandwidth. Levels of subharmonic, harmonics and ultraharmonics were then computed by integration of the spectrum on an $8 \mathrm{kHz}$-band around the frequency of interest.

\section{Cavitation threshold}

The cavitation threshold was set by computing the Stable Cavitation Dose (SCD) $[39,40]$. In this study, the SCD was defined as the sum of harmonics 2 to 9 . Then, the SCD was normalized by a reference SCD called $S C D_{\text {ref. }}$ For experiments mimicking thermal necrosis, the $S C D_{\text {ref }}$ was assessed from the signal recorded in the first $50 \mathrm{~ms}$ of the first sonication, before the appearance of cavitation. For experiments mimicking $\mathrm{BBB}$ opening, the $\mathrm{SCD}_{\text {ref }}$ was computed from the signal recorded before the microbubble injection. Cavitation was deemed significant for a SCD over 1.5 times the SCDref, corresponding to at least 2 times the standard deviation of the SCD during the first sonication (50ms). More precisely, it corresponds to 2 times (respectively 4 times) the standard deviation of the SCD signal during the first sonication for thermal necrosis (respectively for BBB opening).

\section{Localizing cavitation activity}

Once cavitation was detected, its inception point was binary localized (inside or outside the skull) by computing the ratios between harmonics or ultraharmonics. Firstly, six harmonic ratios were computed: harmonics $3,4,5,6,7$ and 8 over harmonic 2; and seven ultraharmonic ratios: ultraharmonics $3 / 2,5 / 2,7 / 2,9 / 2,11 / 2,13 / 2$ and $15 / 2$ over subharmonic $1 / 2$. These ratios were computed for BBB opening and thermal necrosis, and for cavitation occurring inside and outside the skull. 
Two configurations were investigated, corresponding to two true conditions (see Table 1): cavitation occurring inside the skull corresponded to the setup described in Fig 2 (left) with the skull bone in place (Fig. 2 left, in brown color) and cavitation occurring outside the skull corresponded to the setup described in Fig 2 (left) with the skull bone removed.

For a given cavitation event, when the harmonic or ultraharmonic ratio was lower than a given value (that is calculated in the result section) it was classified as detected inside the skull. When the ratio was higher than the threshold it was classified as detected outside the skull.

To assess the repeatability of the method, a statistical study was performed. For each configuration (for example 'BBB opening with propagation through a monkey skull' or 'thermal necrosis with free propagation'), the PCD signals were divided into 50 ms subsections with a $50 \%$ overlap. The power spectra were computed for each $50 \mathrm{~ms}$ time window to obtain the harmonics and ultraharmonics ratios against time. BBB openings were carried out on six human skulls (\#1 to \#6).

Finally, we restricted a more thorough analysis to the 4 to 2 harmonic ratios and the $5 / 2$ to $1 / 2$ ultraharmonic ratios. Receiver operating characteristic (ROC) curves were calculated for the binary classifier 'cavitation inside or outside the skull'. Table 1 details all the possibilities for the binary localization test. For example, a 'true positive' case is defined as 'cavitation occurs inside the skull and detected as such', whereas a 'false positive' is described as 'cavitation occurs outside the skull but detected as cavitation inside the skull'. The ROC curves were exploited to identify the most appropriate threshold for the harmonic and ultraharmonic ratios for each scenario and to assess the safety (high sensitivity) and the efficacy (high specificity) related to our binary localization technique.

\begin{tabular}{|c|c|c|}
\hline & \multicolumn{2}{|c|}{ true conditions } \\
\hline & $\begin{array}{l}\text { cavitation inside the } \\
\text { skull }\end{array}$ & $\begin{array}{l}\text { cavitation outside } \\
\text { the skull }\end{array}$ \\
\hline $\begin{array}{c}\text { cavitation located as being inside the skull, i.e. } \\
\text { ratios lower than a given threshold }\end{array}$ & true positive & false positive \\
\hline $\begin{array}{l}\text { cavitation located as being outside the skull, i.e. } \\
\text { ratios higher than a given threshold }\end{array}$ & false negative & true negative \\
\hline
\end{tabular}

Table 1: Outcomes for the binary classifier 'cavitation inside/outside the skull' depending on the true (actual) and predicted (obtained with binary localization technique) conditions.

\section{Results}

\section{Spectrum modification due to cavitation activity}

Spectra obtained during thermal necrosis experiments are presented in Figure 4 for three different scenarios: (a) 'free propagation', (b) 'propagation through the monkey skull' and (c) 'propagation through human skull \#1'. All the spectra presented in Figure 4 were obtained for 4.5MPa PNP sonications. Spectra in grey are examples with no cavitation evidences $\left(S C D<1.5 \times S C D_{\text {ref }}\right)$. Harmonics are seen in the spectra in the absence of cavitation activity. These harmonics can be induced by electronic nonlinearities in the power amplifier and by nonlinear propagation in water. Subharmonic and ultraharmonics, which are the signature of cavitation activity, are not detectable. Compared to the 'no cavitation evidence' cases, the cases which are deemed 'with cavitation' ( $S C D>1.5 \times S C D_{\text {ref }}$ ) 
present the following spectra features: (i) the harmonic level increases slightly, (ii) the subharmonic and ultraharmonics appear and (iii) the broadband level emerges. The increase in broadband noise is particularly visible in the 'free propagation' case. This phenomenon likely occurs when a skull is in front of the PCD, but, because our PCD is not sensitive enough, the acoustic broadband is lost in the electronic noise. While low frequency harmonics remain easily discernible with or without skull in front of the PCD, the level of high frequency harmonics decreases significantly when skulls are in place.

Figure 5 presents the spectra obtained during BBB opening at 0.6MPa PNP. Figure $5(\mathrm{a}-\mathrm{c})$ present outcomes obtained for: (a) free propagation with no skull in place, (b) propagation through the monkey skull flap affixed in front of the PCD, (c) propagation through human skull \#1. Spectra before UCA injection (no microbubble, 0.6MPa PNP) and after the UCA injection (microbubbles, 0.6MPa PNP) are superimposed in gray and black lines, respectively. In any case, the harmonic level significantly increases when microbubbles circulated in vasculature. Table 2 summarizes some harmonic and ultraharmonic ratios extracted from these spectra.
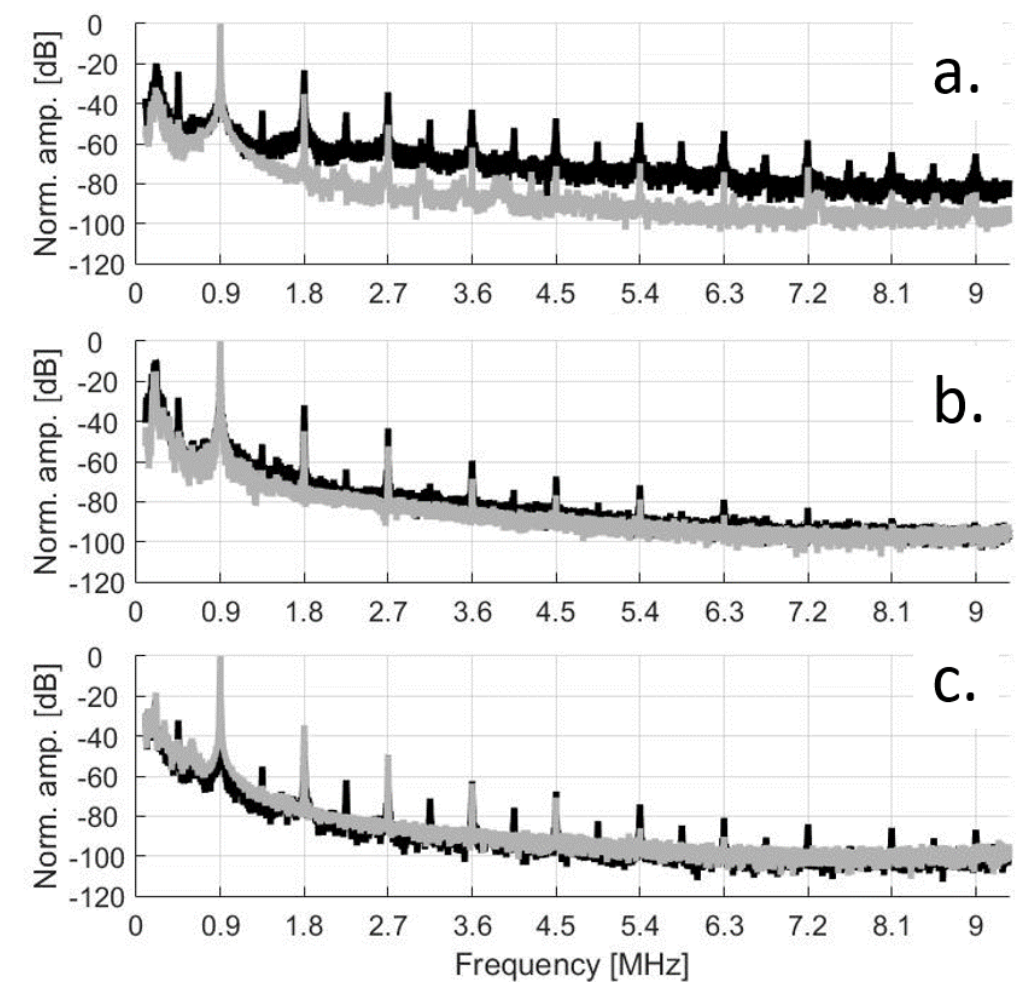

Figure 4: Spectra in decibels obtained from data recorded during thermal necrosis. The grey line is the baseline (4.5MPa PNP sonication, $\mathrm{SCD}<1.5 \times \mathrm{SCD}_{\text {ref }}$ ) and the black one is the cavitation signal (4.5MPa $P N P$ sonication, $\mathrm{SCD}>1.5 \times \mathrm{SCD}_{\text {ref }}$ ). From top to bottom : (a) free propagation, (b) propagation through the monkey skull and (c) propagation through human skull \#1. 

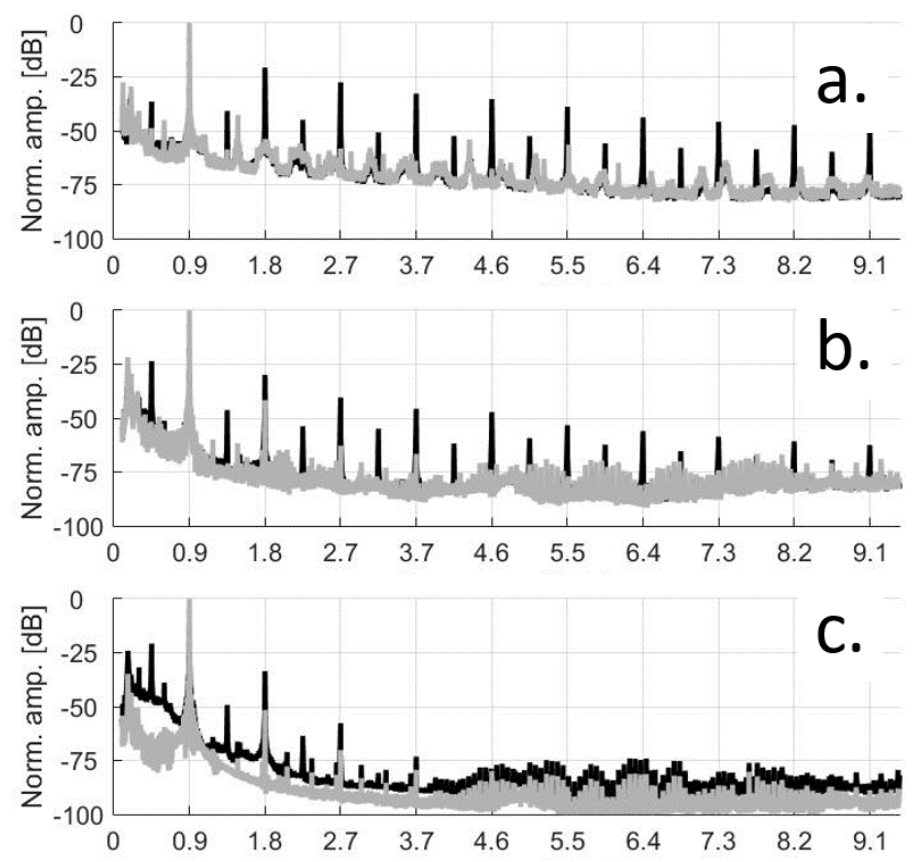

Figure 5: Spectra in decibels obtained from data recorded during $B B B$ openings. The grey line is the baseline (0.6MPa PNP, before UCA injection, $\mathrm{SCD}<1.5 \times \mathrm{SCD}_{\text {ref }}$ ) and the black one is the cavitation signal (0.6MPa PNP, after UCA injection, $\mathrm{SCD}>1.5 \times \mathrm{SCD}_{\text {ref }}$ ). From top to bottom : (a) free propagation,

(b) propagation through the monkey skull and (c) propagation through human skull \#1.

\begin{tabular}{|c|c|c|c|}
\cline { 2 - 4 } \multicolumn{1}{c|}{} & $\begin{array}{c}\text { Free } \\
\text { propagation }\end{array}$ & $\begin{array}{c}\text { Propagation through } \\
\text { the monkey skull }\end{array}$ & $\begin{array}{c}\text { Propagation through } \\
\text { the human skull \#1 }\end{array}$ \\
\hline harmonic ratio & -13 & -16 & -39 \\
\hline ultraharmonic ratio & -7 & -31 & -43 \\
\hline
\end{tabular}

Table 2: Harmonic (harmonics 4 over 2) and ultraharmonic (ultraharmonic $5 / 2$ over subharmonic $1 / 2$ ) ratios in $d B$ for $B B B$ opening.

\section{Harmonic and ultraharmonic ratio}

The harmonic and ultraharmonic ratios were assessed to quantify the modification of the cavitationbased acoustic signal after passing through the skull. Figure 6(a-b) illustrate outcomes from experiments mimicking thermal necrosis. The ratios of ultraharmonics $(3 / 2,5 / 2,7 / 2 \ldots)$ to the $1 / 2$ subharmonic are displayed in Figure $6(a)$. The ratios of harmonics $(3,4,5 \ldots)$ to the harmonic 2 are plotted in Figure 6(b). Three scenarios are displayed: 'free propagation' (red), 'propagation through the monkey skull' (dark blue) and 'propagation through human skull \#1' (light blue).

During BBB opening experiments, similar results were obtained for ultraharmonic (Fig. 6(c)) and harmonic ratios (Fig. 6(d)). Four setups are compared: 'free propagation with no skull in place' (red), 'propagation through the monkey skull sample affixed in front of the PCD' (dark blue) and 'propagation through human skull sample \#1' (light blue). Figure 7 compiles the outcomes from BBB openings on the six human skulls (from \#1 to \#6). These results are compared with the propagation in free water. Throughout our experiments, harmonics and ultraharmonics ratios decrease when ultrasound crosses 
skulls. Consequently, these ratios prove effective to localize cavitation activity inside or outside the skull.
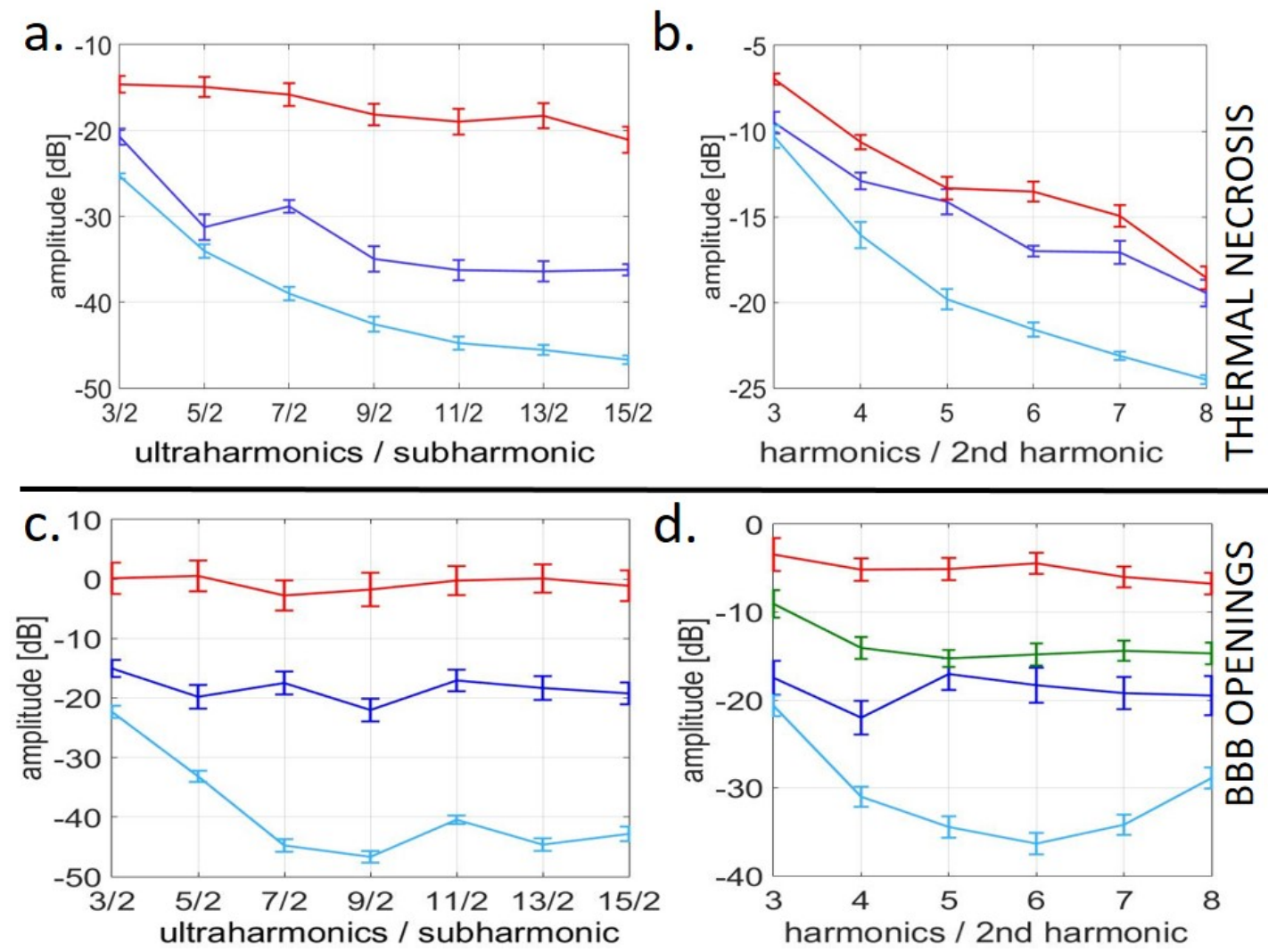

Figure 6: Amplitude in Decibels of ultraharmonic ratios, from ultraharmonic $3 / 2$ to subharmonic 1/2, to ultraharmonic 15/2 to subharmonic 1/2 for thermal necrosis (a) and for BBB opening (c) and harmonic ratios from harmonic 3 over harmonic 2, to harmonic 8 over harmonic 2 during thermal necrosis (b) and during BBB opening (d). Three scenarios are depicted: 'free propagation in water' (red), 'propagation through monkey skull' (dark blue) and 'propagation through human skull \#1' (light blue). Error bars display standard deviation computed over time (signal is divided into 50ms intervals).
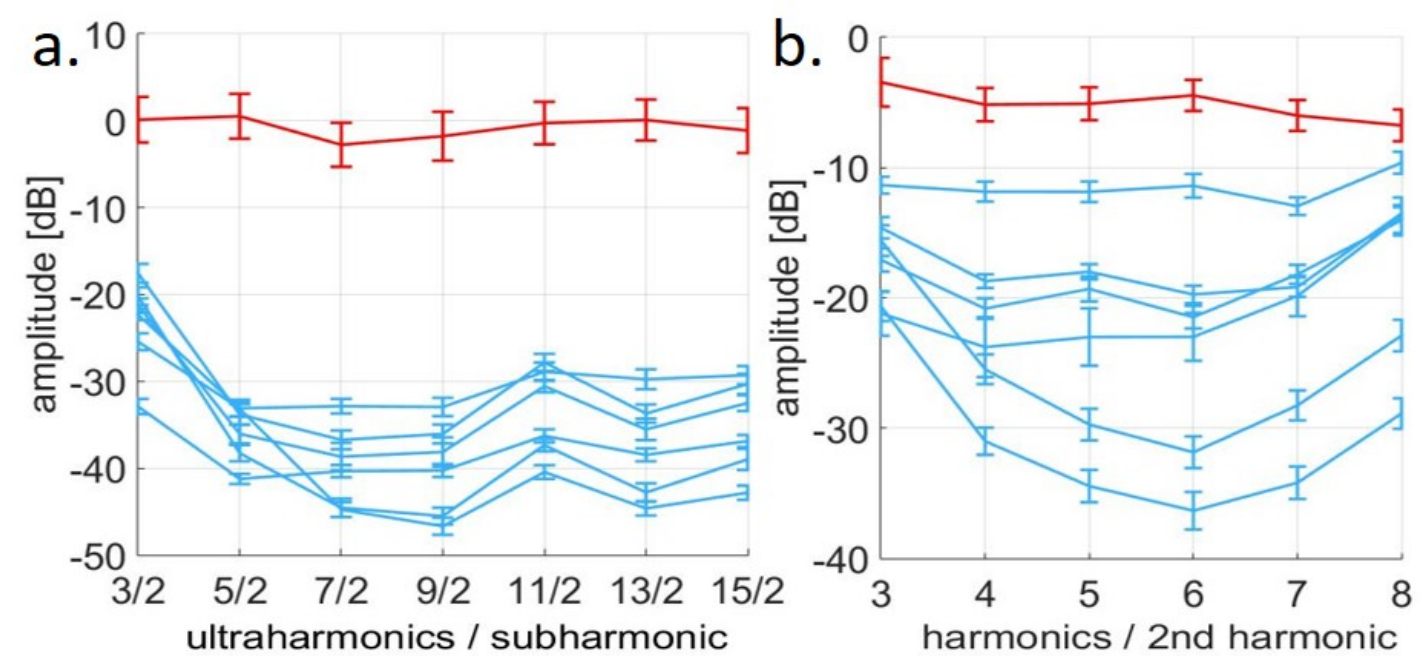
Figure 7: Ultraharmonics (a) and harmonics (b) ratios for BBB openings on six human skulls from \#1 to \#6 (light blue). The 'free propagation in water' case is displayed in red for comparison.

\section{$\underline{\text { Repeatability test }}$}

In this section, the study is restricted to the harmonic 4 to harmonic 2 ratio and ultraharmonic $5 / 2$ to $1 / 2$ ratio.

Figure 8 presents these ratios against time for thermal necrosis. For each case (free propagation, propagation through human skull \#1 and propagation through the monkey skull), 233 measurements of cavitation signal were recorded (within a total of 6 seconds of recording time, 1 measure every $25 \mathrm{~ms})$. The solid lines depict the associated means and the dashed lines the standard deviation.

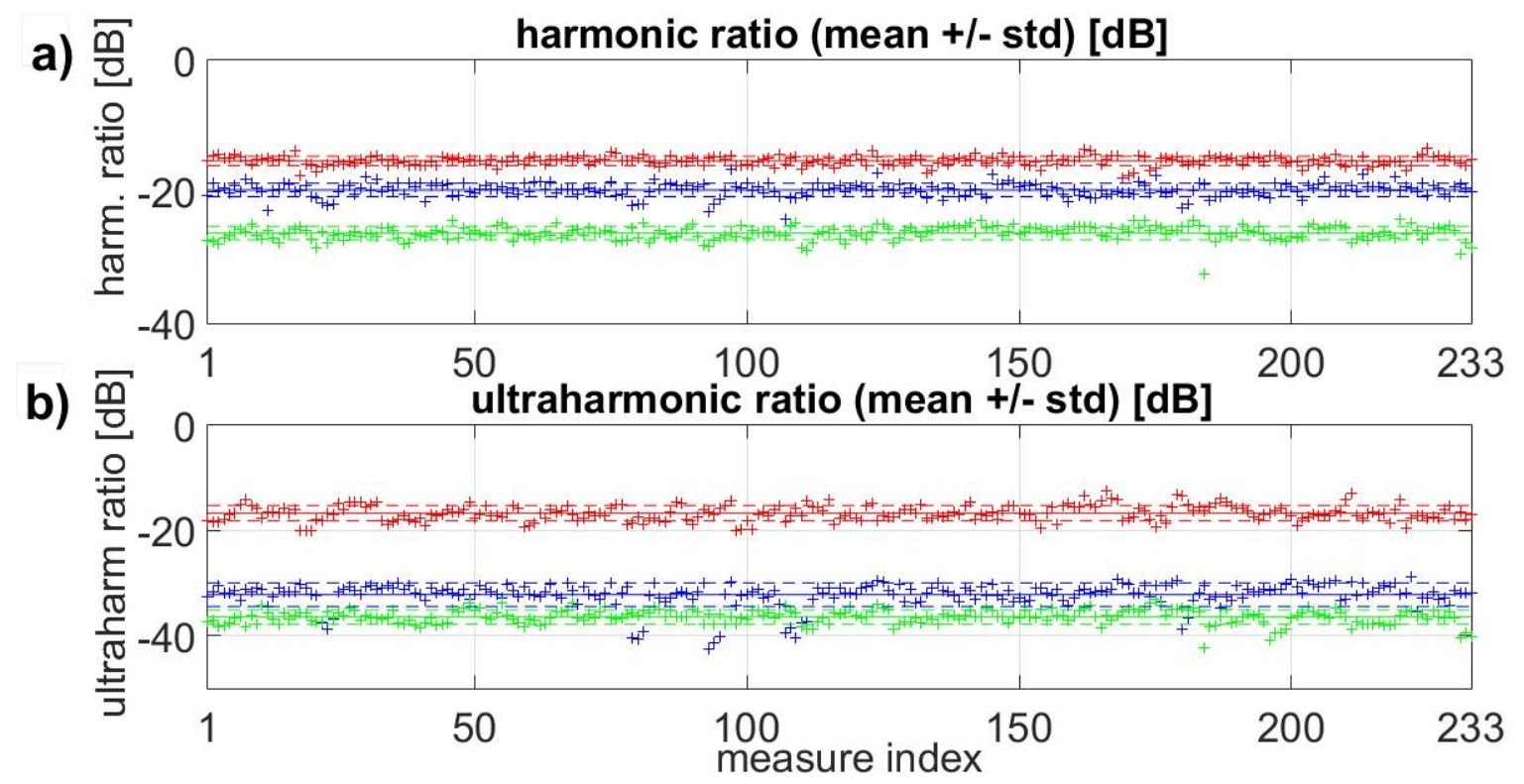

Figure 8: Harmonic 4 to 2 and ultraharmonic $5 / 2$ to $1 / 2$ ratios during thermal experiment in calf brain (with 4.5MPa PNP at focus). Mean (solid line) and standard deviation (dashed lines) are shown. 'Cavitation outside the skull' case is plotted in red, 'cavitation inside the monkey skull' in blue and 'cavitation inside human skull \#1' in green.

Basically, Figure 8 highlights that these ratios are separated into several distinct bands. Namely, the ratios are higher when no skull intercepts the cavitation signal. In order to identify a threshold that could discriminate between one case and another, we computed the ROC curves: the true positive rate was plotted against the false positive rate at various threshold settings. Figure 9 presents the ROC curve associated with the binary localization of microbubbles inside or outside the skull for both thermal necrosis (above) and BBB opening (below). These curves are plotted for the harmonic 4 to 2 ratio (left) and for the ultraharmonic $5 / 2$ to $1 / 2$ ratio (right). The blue line corresponds to 'propagation through the monkey skull compared to free propagation' and the dashed red line to the 'propagation through human skull \#1 compared to free propagation' case. 

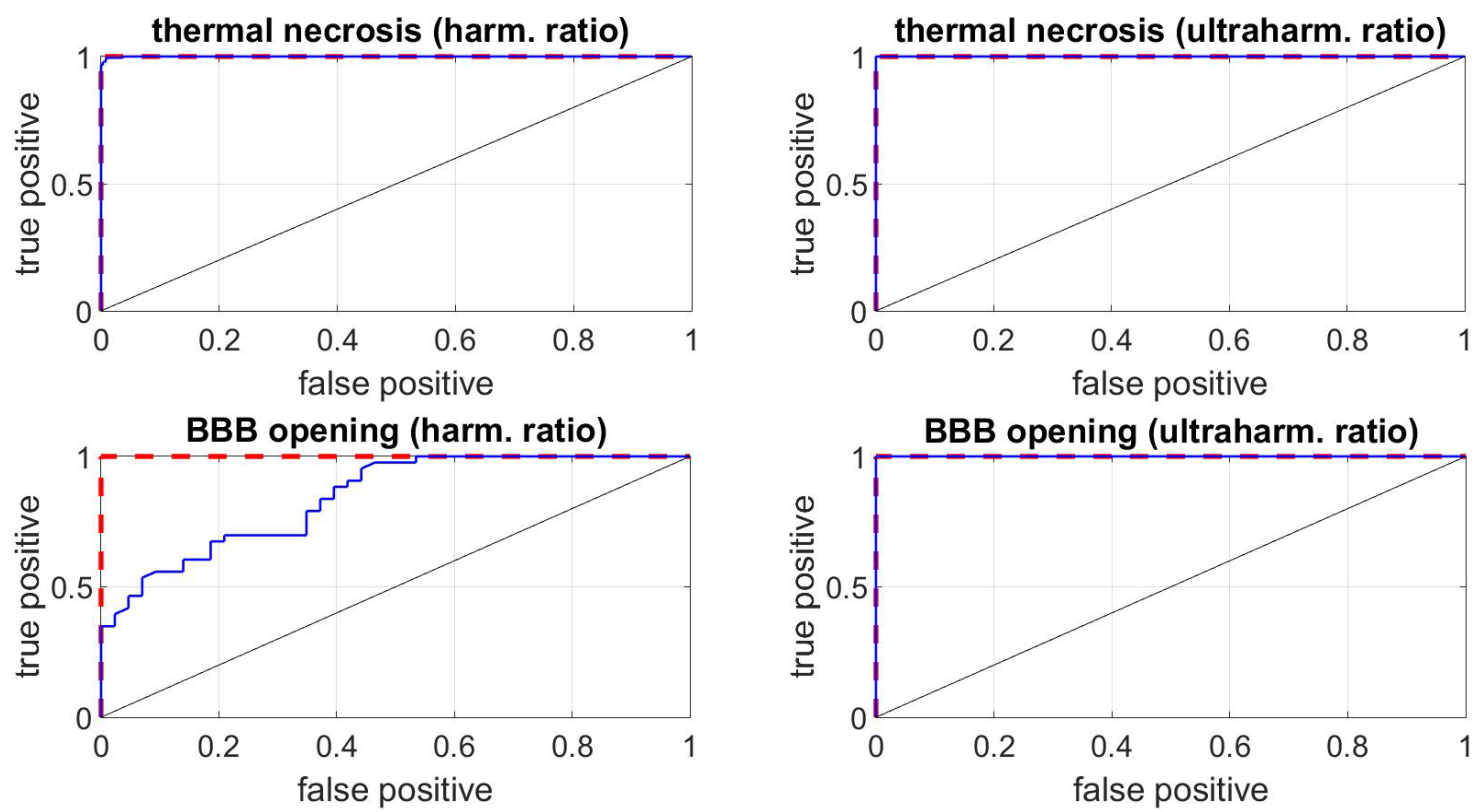

Figure 9: ROC curves of the inside/outside binary localization method for thermal necrosis (top row) and $B B B$ opening (bottom row). Curves are plotted for the harmonic ratio 4 to 2 ratio (left) and for ultraharmonic 5/2 to $1 / 2$ ratio (right). Three cases are plotted: 'propagation through the human skull \#1 compared to free propagation' (dashed red line) and 'propagation through the monkey skull compared to free propagation' (blue line).

Table 3 compiles the sensitivity and specificity obtained from the ROC curves. For each case, we evaluated the threshold providing the best specificity (the largest proportion of true negatives, i.e. the largest proportion of outside cavitation observed as such) for a given sensitivity of $100 \%$ and $99 \%$. The sensitivity corresponds to the proportion of true positives, i.e. the proportion of internal cavitation detected as such.

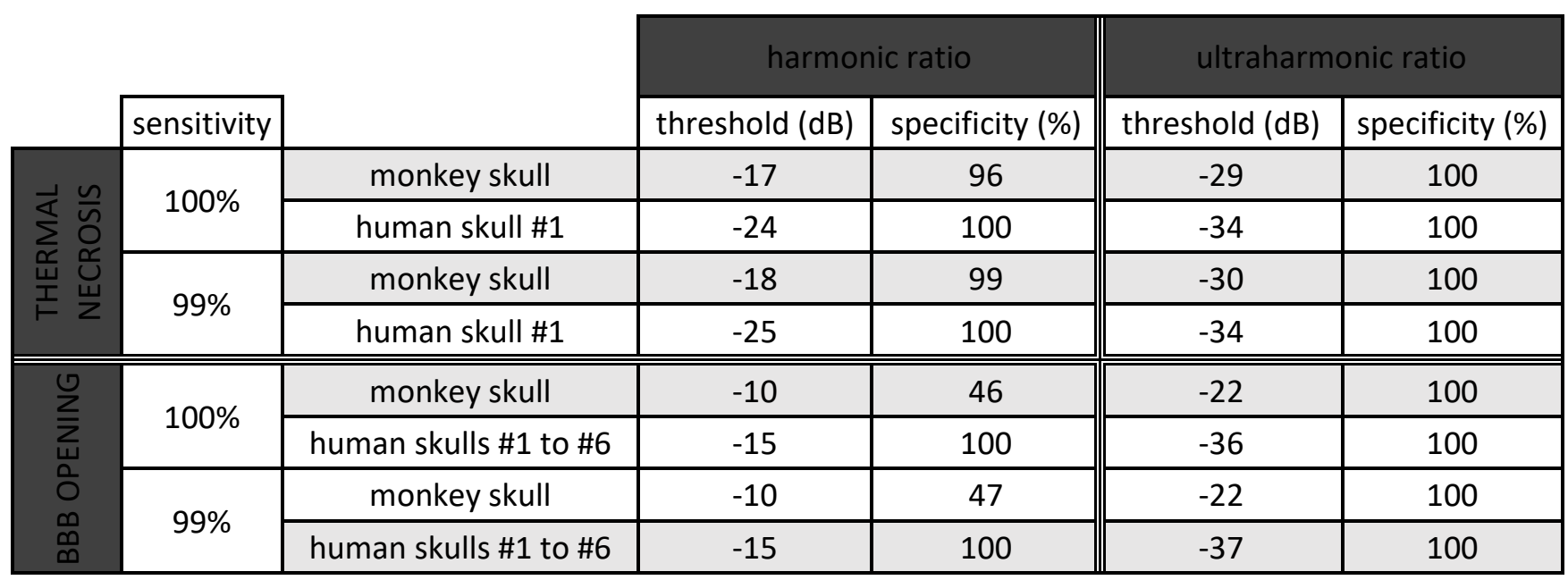

Table 3: Synoptic view of the specificity obtained for a sensitivity of $100 \%$ and $99 \%$, and the corresponding thresholds. Results are obtained for BBB opening and thermal necrosis for the harmonic 4 and the subharmonic 5/2 ratio. 


\section{Discussion}

Spectra acquired during thermal necrosis at 4.5MPa PNP (Fig. 4) confirm that the microbubble nucleation in the calf brain sample is associated with a distinctive acoustic signature containing an increase in harmonic and broadband levels, as well as the emergence of the sub and the ultraharmonics [51]. These results indicate that cavitation occurred in the sample. As hypothesized in our study, the skull was shown to preferentially filter high frequencies: when crossing a human skull, the acoustic signal was attenuated by $-3 \mathrm{~dB}$ for the $1 / 2$ subharmonic -corresponding to $457 \mathrm{kHz}-$ compared to $-19 \mathrm{~dB}$ for the $5 / 2$ ultraharmonic - corresponding to $2.3 \mathrm{MHz}-$. The signal even falls below the electronic noise level beyond the $7^{\text {th }}$ harmonic (i.e for frequencies higher than $6.4 \mathrm{MHz}$ ). The acoustic signals recorded with BBB opening setup after microbubble injection present similar features (Fig. 5): a rise in the harmonic levels and the emergence of the sub and ultraharmonics.

Cavitation is known to be a random phenomenon [51]. The robustness of the method thus needs to be tested. Figures 7 and 8 provide such assessment. The transcranial plots significantly differ from the free water plots, especially for human skull. The monkey BBB data only partially overlap with the free water, and for the harmonic ratio only.

We have demonstrated the possibility to binary discriminate the location of cavitation events, based on the passive recording of harmonic and subharmonic ratios of the cavitation signals. The ROC curves (Fig. 9) showed $100 \%$ sensitivity and $100 \%$ specificity, obtained for all tests carried out with human skulls. The sensitivity and specificity remain equally high when using harmonic or ultraharmonic ratios (Table 3). The method developed here is thus particularly suited for transcranial applications on humans.

For monkey skulls, $100 \%$ sensitivity can be obtained, together with $96 \%$ specificity for the harmonic ratio and $100 \%$ specificity for the ultraharmonic ratio, during thermal necrosis. For BBB opening, results show that the ultraharmonic ratio is more effective than the harmonic ratio: it can achieve $100 \%$ sensitivity and $100 \%$ specificity ( $100 \%$ sensitivity and $46 \%$ specificity for the harmonic ratio). Such a result does not necessarily jeopardize the translation of the harmonic ratio based technique to clinical BBB opening: as mentioned earlier, the harmonic ratio provided $100 \%$ sensitivity and $100 \%$ specificity for BBB openings on humans (as opposed to $100 \%$ sensitivity and $46 \%$ specificity in the BBB monkey setup). This is likely due to the fact that the human skull is thicker than the monkey skull. Consequently, its filtering effect is also stronger. Further investigations are required to assess the efficacy of this binary localization in vivo.

To conclude, despite the random nature of cavitation, the technique is efficient and reliable. Our outcomes indicate that the localization threshold is hardly dependent on the subject. Indeed, the same threshold allowed an effective discrimination (both $100 \%$ sensitivity and specificity) of the cavitation location during thermal necrosis and BBB openings on all the human skulls tested (\#1 to \#6).

This approach is complementary to existing techniques (or others under development) to localize cavitation by active and/or passive mapping in water and gels [44-47, 50]. The efficiency of cavitation mapping was also demonstrated in vitro [49], and in vivo during BBB opening [48] on non human primates, but the integration of imaging array probes remains challenging in clinics. The method introduced in this article provides only binary information about cavitation location, but it is based on a single element PCD. The device is thus inexpensive, compact and easy to install. In addition, existing brain therapy devices already feature individual PCDs[36]. Therefore, upgrading existing devices with this approach implies only a low-cost software modification, and regulatory approval. For clinical use, the PCD could be embedded on the HIFU array to limit its bulk. 
Phase 1 transcranial neurosurgery trials with the Exablate Neuro ${ }^{\circledR}$ system use 8 passive cavitation sensors to detect cavitation and halt the treatment if cavitation is detected [55]. In general, during transcranial thermal ablation, cavitation can occur either inside the brain or in the coupling medium between the skin and the transducer. Different courses of action could be taken to avoid cavitation during the next sonication, depending on whether the microbubbles appeared inside or outside the brain. On the one hand, if cavitation appeared inside the brain, the acoustic power can be lowered and the sonication time increased. On the other hand, if cavitation occurs in the coupling water, the medical staff has the opportunity to perform a more advanced degassing of the coupling water before attempting sonication again, with the same acoustic parameters. The Exablate Neuro ${ }^{\circledR}$ system does not provide information about the inside/outside localization of the cavitation events. Nevertheless, it comprises an efficient degazing system and the aperture of the transducer has been set to the largest possible (a hemisphere) in order to distribute the energy over a large surface of the skin and provide a high antenna gain at focus. Cavitation, if any, is thus very likely to take place in the brain and not in the coupling medium. Cavitation is more likely to occur in the coupling water, as well as in the brain, in devices with a lower aperture like the 512 -element $1 \mathrm{MHz}$ prototype developed at Institut Langevin $[12,56]$. A direct extension of the proof of concept introduced in this paper would be to test this approach with such a preclinical setup [12].

One particular type of disambiguation was not investigated here but could potentially have a major clinical interest: ultrasound induced cavitation in the brain (with or without contrast agents) as opposed to cavitation signal originating from existing bubbles trapped in the coupling medium during the filling procedure, outside the head. Microscopic as well as macroscopic bubbles can indeed be trapped in the coupling medium either during BBB opening or during thermal ablation. The acoustic signatures of such trapped bubbles would need to be recorded and compared to the harmonic content of ultrasound induced cavitation inside the brain. This is beyond the scope of the proof of concept introduced in this paper but could be tested, preferably with a more clinically realistic setup, such as the $1 \mathrm{MHz}$ prototype $[12]$ or the Exablate Neuro ${ }^{\circledR}[31-33,57]$ with a head phantom [58].

In a clinical setup, microbubbles could potentially appear both inside and outside the skull, making the method inoperative. However, if the detection device reacts to the first occurrence of cavitation, the method could remain efficient clinically. Indeed, cavitation is very unlikely to occur simultaneously in two different locations. To be efficient, the system has to react as soon as the first bubbles appear. All data were processed offline in this study. The hardware could be upgraded to perform real time measurements and computation. A $10 \mathrm{~ms}$ reaction time would be the goal, since sonication has to be halted before large bubbles form and can induce potentially serious side effects [59, 60]. FFTs of the cavitation signal could be computed fast to obtain the harmonic and ultraharmonic ratios, as demonstrated by Chettab et al. [61], who assessed a cavitation index every $10 \mathrm{~ms}$ for real-time control of cavitation by computing such FFTs.

\section{Conclusion}

By taking advantage of the low-pass filtering effect induced by the skull, this study demonstrates that a binary localization of microbubble activity inside (i.e. in the brain) or outside (i.e. in the coupling water or in the scalp) the head can be achieved with a single PCD transducer. The ratio between highfrequency harmonics and low-frequency harmonics computed from the acoustic response of microbubbles is indeed significantly modified when crossing a skull (human or monkey). The statistical study of our binary localization tests shows that this approach is relevant for both BBB opening and thermal necrosis. The sensitivity and specificity of these tests were evaluated and provided satisfactory 
outcomes, especially for the ultraharmonic ratio, for which both aforementioned parameters are equal to $100 \%$ in human and monkey. A similar assessment was performed for the harmonic ratio. Results remain perfect for human ( $100 \%$ sensitivity and specificity) but providing mixed outcomes for monkeys due to the lower attenuation compared to a human skull. Further developments are needed to assess this localization's method in vivo and in vitro using a clinical setup such as the Exablate Neuro ${ }^{\circledR}$ (Insightec, Israel). Nevertheless, these results are already promising to offer a reliable and real-time localization of cavitation activity during transcranial focused ultrasound operations.

\section{Acknowledgments}

This work was supported by the Bettencourt Schueller Foundation and the "Agence Nationale de la Recherche" under the program "Future Investments" with the reference ANR-10-EQPX-15.

\section{$\underline{\text { References }}$}

1. McDannold, N., et al., Temporary disruption of the blood-brain barrier by use of ultrasound and microbubbles: safety and efficacy evaluation in rhesus macaques. Cancer research, 2012. 72(14): p. 3652-3663.

2. Barnard, J.W., et al., Effects of high intensity ultrasound on the central nervous system of the cat. Journal of Comparative Neurology, 1955. 103(3): p. 459-484.

3. Fry, W.J. and R. Meyers, Ultrasonic method of modifying brain structures. Confin Neurol, 1962. 22: p. 315-27.

4. Tanter, M., J.-L. Thomas, and M. Fink, Focusing and steering through absorbing and aberrating layers: application to ultrasonic propagation through the skull. The Journal of the Acoustical Society of America, 1998. 103(5): p. 2403-2410.

5. Misra, A., et al., Drug delivery to the central nervous system: a review. J Pharm Pharm Sci, 2003. 6(2): p. 252-73.

6. Sun, J. and K. Hynynen, The potential of transskull ultrasound therapy and surgery using the maximum available skull surface area. J Acoust Soc Am, 1999. 105(4): p. 2519-27.

7. Hynynen, K. and J. Sun, Trans-skull ultrasound therapy: the feasibility of using image-derived skull thickness information to correct the phase distortion. IEEE Trans Ultrason Ferroelectr Freq Control, 1999. 46(3): p. 752-5.

8. Miller, G.W., et al., Ultrashort echo-time MRI versus CT for skull aberration correction in MRguided transcranial focused ultrasound: In vitro comparison on human calvaria. Medical physics, 2015. 42(5): p. 2223-2233.

9. Wintermark, M., et al., T1-weighted MRI as a substitute to CT for refocusing planning in MRguided focused ultrasound. Physics in medicine and biology, 2014. 59(13): p. 3599.

10. Aubry, J.F., et al., Experimental demonstration of noninvasive transskull adaptive focusing based on prior computed tomography scans. J Acoust Soc Am, 2003. 113(1): p. 84-93.

11. Clement, G. and K. Hynynen, A non-invasive method for focusing ultrasound through the human skull. Physics in Medicine and Biology, 2002. 47: p. 1219-1236.

12. L. Marsac, D.C., R. La Greca, A-L Boch, K. Chaumoitre, M. Tanter, J-F Aubry, Ex vivo optimization of a heterogeneous speed of sound model of the human skull for non-invasive transcranial focused ultrasound at $1 \mathrm{MHz}$. International journal of hyperthermia, 2017.

13. Pulkkinen, A., et al., Simulations and measurements of transcranial low-frequency ultrasound therapy: skull-base heating and effective area of treatment. Physics in Medicine and Biology, 2011. 56(15): p. 4661.

14. Haluska, M. and M.L. Anthony, Osmotic blood-brain barrier modification for the treatment of malignant brain tumors. Clinical journal of oncology nursing, 2004. 8(3). 
15. Hamstra, D.A., et al., Intratumoral injection of BCNU in ethanol (DTI-015) results in enhanced delivery to tumor-a pharmacokinetic study. Journal of neuro-oncology, 2005. 73(3): p. 225238.

16. Westphal, M., et al., Gliade ${ }^{\circledR}$ wafer in initial surgery for malignant glioma: long-term followup of a multicenter controlled trial. Acta neurochirurgica, 2006. 148(3): p. 269-275.

17. Rapoport, S.I., Osmotic opening of the blood-brain barrier: principles, mechanism, and therapeutic applications. Cellular and molecular neurobiology, 2000. 20(2): p. 217-230.

18. Kongkham, P.N., et al., Complications in 622 cases of frame-based stereotactic biopsy, a decreasing procedure. The Canadian Journal of Neurological Sciences, 2008. 35(01): p. 79-84.

19. Hynynen, K., et al., Non-invasive opening of BBB by focused ultrasound. Acta Neurochir Suppl, 2003. 86: p. 555-8.

20. Choi, J.J., et al., Noninvasive and transient blood-brain barrier opening in the hippocampus of Alzheimer's double transgenic mice using focused ultrasound. Ultrason Imaging, 2008. 30(3): p. $189-200$.

21. Kinoshita, M., et al., Noninvasive localized delivery of Herceptin to the mouse brain by MRIguided focused ultrasound-induced blood-brain barrier disruption. Proceedings of the National Academy of Sciences, 2006. 103(31): p. 11719-11723.

22. Nance, E., et al., Non-invasive delivery of stealth, brain-penetrating nanoparticles across the blood- brain barrier using MRI-guided focused ultrasound. Journal of Controlled Release, 2014. 189: p. 123-132.

23. Marquet, F., et al., Real-time, transcranial monitoring of safe blood-brain barrier opening in non-human primates. PloS one, 2014. 9(2): p. e84310.

24. Phelps, A. and T. Leighton. 'Acoustic bubble sizing using two frequency excitation techniques. in Proceedings of the 2nd European Conference on Underwater Acoustics. 1994.

25. Pernot, M., et al., In vivo transcranial brain surgery with an ultrasonic time reversal mirror. J Neurosurg, 2007. 106(6): p. 1061-6.

26. Dervishi, E., et al., Transcranial high intensity focused ultrasound therapy guided by 7 TESLA $M R I$ in a rat brain tumour model: A feasibility study. International Journal of Hyperthermia, 2013. 29(6): p. 598-608.

27. Marquet, F., et al., Non-invasive ultrasonic surgery of the brain in non-human primates. The Journal of the Acoustical Society of America, 2013. 134(2): p. 1632-1639.

28. Hynynen, K., et al., Pre-clinical testing of a phased array ultrasound system for MRI-guided noninvasive surgery of the brain--a primate study. European Journal of Radiology, 2006. 59(2): p. 149-56.

29. Hynynen, K., et al., 500-element ultrasound phased array system for noninvasive focal surgery of the brain: a preliminary rabbit study with ex vivo human skulls. Magnetic Resonance in Medicine, 2004. 52(1): p. 100-7.

30. Huss, D.S., et al., Functional assessment and quality of life in essential tremor with bilateral or unilateral DBS and focused ultrasound thalamotomy. Movement Disorders, 2015. 30(14): p. 1937-1943.

31. Lipsman, N., et al., MR-guided focused ultrasound thalamotomy for essential tremor: a proofof-concept study. The Lancet Neurology, 2013. 12(5): p. 462-468.

32. Chang, J.W., et al., Neurophysiologic correlates of sonication treatment in patients with essential tremor. Ultrasound in medicine \& biology, 2015. 41(1): p. 124-131.

33. Elias, W.J., et al., A Pilot Study of Focused Ultrasound Thalamotomy for Essential Tremor. New England Journal of Medicine, 2013. 369(7): p. 640-648.

34. Magara, A., et al., First experience with MR-guided focused ultrasound in the treatment of Parkinson's disease. Journal of therapeutic ultrasound, 2014. 2(1): p. 1.

35. Martin, E., et al., High-intensity focused ultrasound for noninvasive functional neurosurgery. Ann Neurol, 2009. 66(6): p. 858-61. 
36. Jeanmonod, D., et al., Transcranial magnetic resonance imaging-guided focused ultrasound: noninvasive central lateral thalamotomy for chronic neuropathic pain. Neurosurgical Focus, 2012. 32(1): p. 1-11.

37. Jung, H., et al., Bilateral thermal capsulotomy with MR-guided focused ultrasound for patients with treatment-refractory obsessive-compulsive disorder: a proof-of-concept study. Molecular psychiatry, 2014.

38. $\mathrm{Xu}, \mathrm{Z}$., et al., Intracranial inertial cavitation threshold and thermal ablation lesion creation using MRI-guided 220-kHz focused ultrasound surgery: preclinical investigation. Journal of neurosurgery, 2014: p. 1-10.

39. McDannold, N., N. Vykhodtseva, and K. Hynynen, Targeted disruption of the blood-brain barrier with focused ultrasound: association with cavitation activity. Physics in medicine and biology, 2006. 51(4): p. 793-807.

40. Wu, S.-Y., et al., Transcranial cavitation detection in primates during blood-brain barrier opening-a performance assessment study. Ultrasonics, Ferroelectrics, and Frequency Control, IEEE Transactions on, 2014. 61(6): p. 966-978.

41. Tu, J., et al., Intravascular inertial cavitation activity detection and quantification in vivo with Optison. Ultrasound Med Biol, 2006. 32(10): p. 1601-9.

42. O'Reilly, M.A. and K. Hynynen, Blood-brain barrier: real-time feedback-controlled focused ultrasound disruption by using an acoustic emissions-based controller. Radiology, 2012. 263(1): p. 96-106.

43. Tung, Y.S., et al., In vivo transcranial cavitation threshold detection during ultrasound-induced blood-brain barrier opening in mice. Phys Med Biol, 2010. 55(20): p. 6141-55.

44. Salgaonkar, V.A., et al., Passive cavitation imaging with ultrasound arrays. The Journal of the Acoustical Society of America, 2009. 126(6): p. 3071-3083.

45. Farny, C.H., R.G. Holt, and R.A. Roy, Temporal and spatial detection of HIFU-induced inertial and hot-vapor cavitation with a diagnostic ultrasound system. Ultrasound in medicine \& biology, 2009. 35(4): p. 603-615.

46. Gyongy, M. and C.C. Coussios, Passive cavitation mapping for localization and tracking of bubble dynamics. The Journal of the Acoustical Society of America, 2010. 128(4): p. EL175-80.

47. Gyongy, M. and C.C. Coussios, Passive spatial mapping of inertial cavitation during HIFU exposure. IEEE Trans Biomed Eng, 2009. 57(1): p. 48-56.

48. Arvanitis, C.D., G.T. Clement, and N. McDannold, Transcranial assessment and visualization of acoustic cavitation: modeling and experimental validation. Medical Imaging, IEEE Transactions on, 2015. 34(6): p. 1270-1281.

49. Arvanitis, C.D. and N. McDannold, Integrated ultrasound and magnetic resonance imaging for simultaneous temperature and cavitation monitoring during focused ultrasound therapies. Medical physics, 2013. 40(11): p. 112901.

50. Gateau, J., et al., Combined passive detection and ultrafast active imaging of cavitation events induced by short pulses of high-intensity ultrasound. Ultrasonics, Ferroelectrics and Frequency Control, IEEE Transactions on, 2011. 58(3): p. 517-532.

51. Leighton, T., The acoustic bubble. 1994: Academic press.

52. Fry, F.J. and J.E. Barger, Acoustical properties of the human skull. The Journal of the Acoustical Society of America, 1978. 63(5): p. 1576-1590.

53. Barriere, C. and D. Royer, Optical measurement of large transient mechanical displacements. Applied Physics Letters, 2001. 79(6): p. 878-880.

54. McDannold, N., N. Vykhodtseva, and K. Hynynen, Blood-brain barrier disruption induced by focused ultrasound and circulating preformed microbubbles appears to be characterized by the mechanical index. Ultrasound in medicine \& biology, 2008. 34(5): p. 834-40.

55. Jung, H.H., et al., Different magnetic resonance imaging patterns after transcranial magnetic resonance-guided focused ultrasound of the ventral intermediate nucleus of the thalamus and anterior limb of the internal capsule in patients with essential tremor or obsessivecompulsive disorder. Journal of neurosurgery, 2015. 122(1): p. 162-168. 
56. Aubry, J.-F., et al., High intensity focused ultrasound for transcranial therapy of brain lesions and disorders. IRBM, 2010. 31(EPFL-ARTICLE-188661): p. 87-91.

57. Eames, M.D., et al., Trans-cranial focused ultrasound without hair shaving: feasibility study in an ex vivo cadaver model. Journal of Therapeutic Ultrasound, 2014. 1(1): p. 1-6.

58. Eames, M.D., et al., Head phantoms for transcranial focused ultrasound. Medical physics, 2015. 42(4): p. 1518-1527.

59. Chen, W.-S., et al., Inertial cavitation dose and hemolysis produced in vitro with or without Optison $^{\circledR}$. Ultrasound in medicine \& biology, 2003. 29(5): p. 725-737.

60. Hwang, J.H., et al., Correlation between inertial cavitation dose and endothelial cell damage in vivo. Ultrasound in medicine \& biology, 2006. 32(10): p. 1611-1619.

61. Chettab, K., et al., Spatial and Temporal Control of Cavitation Allows High In Vitro Transfection Efficiency in the Absence of Transfection Reagents or Contrast Agents. PloS one, 2015. 10(8): p. e0134247. 